Die Autoren fanden in keinem der Punkte Unterschiede zwischen den beiden Gruppen: Sowohl hinsichtlich der Häufigkeit der ADHS-Diagnose, der ADHSSymptome, der Komorbiditäten, ADHS in der Familie und der funktionalen Beeinträchtigungen waren die im September Geborenen und damit später eingeschulten Kinder mit ADHS den AugustKindern gleich.

Die Autoren schließen aus ihrer Untersuchung, dass der Zeitpunkt des Schuleintritts weder einen Einfluss auf die ADHS-Diagnose noch auf seine Ausprägung hat. Wenn bei einem Schulkind ADHS-Symptome vorhanden seien, so sei die Diagnose „ADHS“ zu stellen und nicht eine „Unreife mit zu früher Einschulung" dafür verantwortlich zu machen. Insbesondere die Tatsache, dass eine familiäre ADHS in beiden Gruppen gleich häufig auftrat, weist auf eine genetische Ursache und nicht auf eine durch Unreife bedingte ADHSSymptomatik hin.

Biederman J et al. Is the diagnosis of ADHD influenced by time of entry to school? An examination of clinical, familial, and functional correlates in children at early and late entry points. J Atten Disord 2012 May 24. [Epub ahead of print]

Kommentar: Diese Arbeit steht im Gegensatz zu der Beobachtung von Morrow et al. (2012), die feststellten, dass in einer Schulklasse die jüngeren Kinder häufiger mit ADHS diagnostiziert werden als die Älteren. Sie vermuten daher, dass die zerebrale Unreife der jüngeren Kinder dazu führt, ihr von den älteren Kindern abweichendes Verhalten mit der Diagnose "ADHS“ zu versehen. Möglicherweise beruht der Unterschied in den Ergebnissen der beiden Autoren darauf, dass in der aktuellen Studie von Biederman die Diagnose von auf ADHS spezialisierten Experten gestellt worden war, während in der Kohortenstudie von Morrow alle Kinder erfasst wurden, die von wem auch immer die Diagnose „ADHS" erhalten hatten.

Was folgt daraus? Die Diagnose "ADHS" sollte auch bei den Klassenjüngsten gestellt werden, wenn sie entsprechend den Leitlinien ADHS die Kriterien erfüllen, insbesondere wenn die Entwicklungsanamnese und die psychosoziale Familienanamnese auf eine ADHS hinweisen. ADHS ist eine genetisch bedingte Störung und nicht die Folge von „zu früher Einschulung“. Dies entspricht auch unserer Beobachtung von Kindern mit der Diagnose "ADHS": dass die ADHS-Symptome auch bei einer um ein Jahr verzögerten Einschulung störungsrelevant persistieren. Es sollte aber darauf geachtet werden, nicht vorschnell Einzelsymptome als ADHS zu diagnostizieren. Besonders wenn in der Familie keine weiteren Mitglieder eine ADHS haben und das Kind bis zur Einschulung unauffällig war, sollte die Diagnose nur mit Vorbehalt gestellt werden.

Dr. Kirsten Stollhoff

\title{
Typ-1-Diabetes in Europa immer häufiger
}

\section{Wie hat sich in Europa die Inzidenz des Typ-1-Diabetes entwickelt? Darauf sollte eine Studie Antwort geben, die die 20-Jahre-Inzidenztrends im Kindes- alter beschreibt. Verglichen wurden die Anstiegsraten von 1989-1998 und von 1999-2008.}

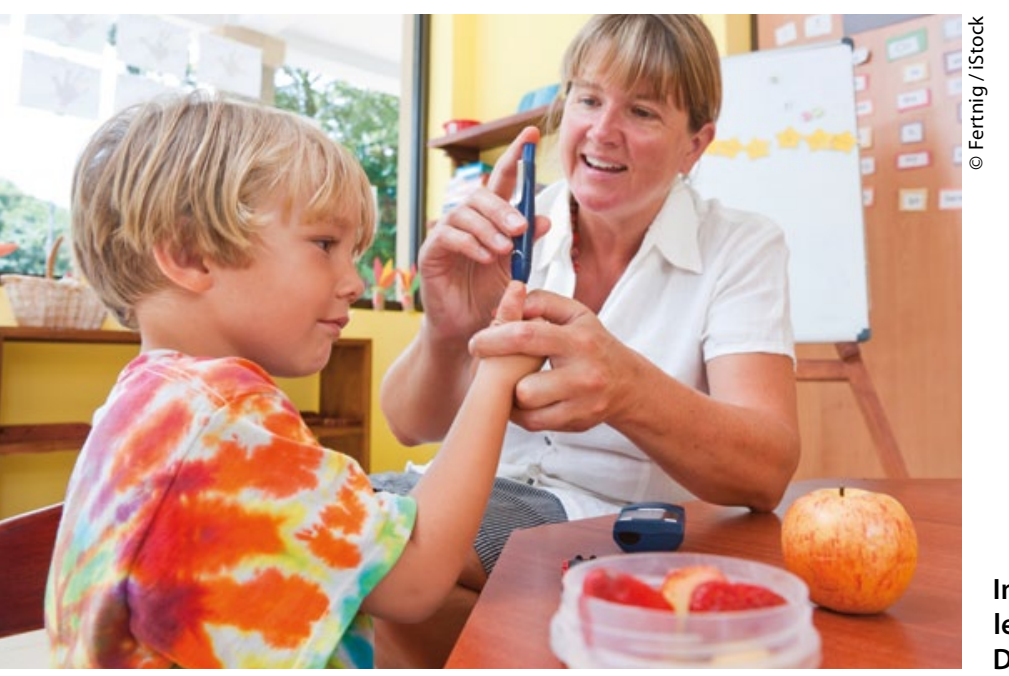

Immer mehr Kinder leiden in Europa an Diabetes.

n der epidemiologischen Studie registrierten 23 europäische Zentren (EURODIAB) aus 19 Ländern 49.969 neue Typ1-Diabetes-Fälle (Diagnose vor dem 15. Geburtstag). Die meisten Register erfass- ten mehr als $90 \%$ der Fälle. Während des 20-Jahres-Zeitraumes war die Inzidenz bis auf eine Ausnahme in allen Registern signifikant verändert: Sie stieg insgesamt an. Die medianen Anstiegsraten der ers- ten und zweiten Zeitspanne lagen bei $3,4 \%$ und 3,3\% pro Jahr. Die Raten für die erste und zweite Periode unterschieden sich bei neun der 21 Registern: Fünf Register zeigten signifikant höhere Raten in der ersten, vier zeigten signifikant höhere Raten in der zweiten Hälfte.

Demnach steigt in Europa die Typ1-Diabetes-Inzidenz im Kindesalter um $3,4 \%$ pro Jahr über einen Verlauf von 20 Jahren an. In einigen Registern gibt es aber Perioden mit langsameren und schnelleren Anstiegen. Das Veränderungsmuster verdeutlicht, dass wichtige Risikofaktoren in den verschiedenen Ländern über die Zeit unterschiedlich Einfluss nehmen müssten.

Patterson CC et al. Trends in childhood type 1 diabetes incidence in Europe during 1989-2008: evidence of non-uniformity over time in rates of increase. Diabetologia 2012; 55: 2142-7

Kommentar: Diese Studie zeigt erneut, wie stark und flächendeckend der Typ-1-Diabetes im Kindesalter zunimmt. Sie legt nahe, dass weit verbreitete Umweltfaktoren zu diesem Anstieg führen müssen. Eine frühe Diagnose ist wichtig, damit sich die Ketoazidoserate nicht parallel erhöht und damit die Gefahr schwerer Komplikationen steigt.

PD Dr. Nanette C. Schloot 